\title{
Lumpy Bumpy Weepy Face - A Rare Case Report of a Frequently Misdiagnosed Disease
}

\author{
Ramachandran Ramakrishnan ${ }^{1}$, Dhivya Sundararajan², Shivasekar Ganapathy³, Balaji Durairaj ${ }^{4}$, Murali Narasimhan ${ }^{5}$ \\ ${ }^{1}$ Department of Dermatology, Venereology and Leprosy, SRM Medical College \& Hospital, Kattankulathur, Potheri, \\ Tamil Nadu, India. ${ }^{2}$ Department of Dermatology, Venereology and Leprosy, SRM Medical College \& Hospital, \\ Kattankulathur, Potheri, Tamil Nadu, India. ${ }^{3}$ Department of Pathology, SRM Medical College \& Hospital, \\ Kattankulathur, Potheri, Tamil Nadu, India. ${ }^{4}$ Department of General Surgery, SRM Medical College \& Hospital, \\ Kattankulathur, Potheri, Tamil Nadu, India. ${ }^{5}$ Department of Dermatology, Venereology and Leprosy, SRM Medical \\ College \& Hospital, Kattankulathur, Potheri, Tamil Nadu, India.
}

\section{INTRODUCTION}

Infectious clinical conditions that can present with swellings and sinuses more commonly, include cutaneous tuberculosis, and subcutaneous and deep fungal infections. One of the differentials that should be considered in such a scenario is actinomycosis. Actinomyces are filamentous gram-positive bacteria that are facultative anaerobes which can form endospores. The individual bacterium is rod shaped filamentous organism. After its discovery in 1890, a misconception was that it is a mycosis that affected individuals who chewed grass or straw. The pathogen is still known as the 'great masquerader'. ${ }^{1}$

Many entities can mimic cutaneous mycobacterial infections and deep cutaneous mycosis. One such great mimicker is actinomycosis - a bacterial infection caused by Actinomyces israelii. Though it is sensitive to many antibiotics, the duration of treatment for effective clearance of the lesions is long.

\section{PRESENTATION OF CASE}

A previously healthy 51-year-old female agricultural worker presented with multiple nodular lesions over the face and scalp with pus discharge from some of the nodules, over a period of 1 year. There was no history of trauma, dental procedures or fever prior to the onset of these lesions. Gradually over the last year, she experienced progression of the lesion in size and number and she felt a vague local pain. Subsequently she developed serous and pus discharge on and off from few lesions. In the past 3 months, she had gradual worsening of restriction in opening her mouth. Past medical history and Family history was negative for significant illness or similar complaints.

On clinical examination, she had multiple, well defined, indurated, hyperpigmented subcutaneous firm nodules (Fig. 1A) over the forehead \& few plaques with a single active discharging sinus (Fig. 1B) in right pre auricular region. Multiple puckered scars (Fig. 1B) were noted on the right pre auricular area \& right side of forehead. Restricted mouth opening (able to insert 2 fingers) was noted. Additionally, she had 0.5 to $1 \mathrm{~cm}$, multiple, tender, firm, discrete and mobile submandibular, sub mental and pre auricular lymph nodes on the right side. Oral mucosa was normal.
Corresponding Author: Dr. Murali Narasimhan, Head of Department, Department of DVL, SRM Medical College and Hospital And Research centre, Potheri, Tamilnadu, India.

E-mail: leecutis@gmail.com

DOI: $10.14260 / \mathrm{jemds} / 2020 / 599$

How to Cite This Article:

Ramakrishnan $R$, Sundararajan $D$, Ganapathy $S$, et al. Lumpy bumpy weepy face - a rare case report of a frequently misdiagnosed disease. J Evolution Med Dent Sci 2020;9(37):2761-2763, DOI: 10.14260/jemds/2020/599

Submission 01-06-2020,

Peer Review 25-07-2020,

Acceptance 03-08-2020,

Published 14-09-2020.

Copyright (C) 2020 JEMDS. This is an open access article distributed under Creative Commons Attribution License [Attribution 4.0 International (CC BY 4.0)] 




DIFFERENTIAL DIAGNOSIS

Scrofuloderma

Atypical Mycobacterial Infection

Actinomycotic Mycetoma

\section{PATHOLOGICAL DISCUSSION}

Bedside staining procedures from the discharge showed inflammatory cells in Gram's stain \& methylene blue stain; negative for fungal elements in $\mathrm{KOH}$ preparation; negative for AFB in Ziehl Nielsen Stain. Complete blood count, liver \& renal function tests, ESR and blood sugar were normal. Mantoux test was negative for induration. Anti - HIV antibody and HBsAg serology were negative and CRP was within normal limits. X Ray of skull showed soft tissue lesion adjacent to ramus of mandible. Culture for fungus, AFB (M. tuberculosis) and bacteria were negative.

An excision biopsy from the lesional area showed an acute - on - chronic inflammatory infiltrate composed of neutrophils, lymphocytes, plasma cells and histiocytes. Colonies of gram-positive filamentous bacteria with Splendore - Hoeppli phenomenon was seen (Fig 1C \& 1D). Findings were consistent with Actinomycosis.

\section{DISCUSSION OF MANAGEMENT}

The patient was started on Tab. Amoxycillin-Clavulanic acid (500 mg + $125 \mathrm{mg}$ ) twice daily and Tab. Cotrimoxazole double strength (sulfamethoxazole $800 \mathrm{mg}+$ trimethoprim $160 \mathrm{mg}$ ) twice daily. The lesions started to resolve during second month of therapy.

\section{DISCUSSION}

Actinomycosis has been called as "the most misdiagnosed disease" even by experienced clinicians and listed as a "rare disease" by the office of rare diseases (ORD) of the National Institute of Health (NIH). ${ }^{2}$ Synonyms include lumpy jaw (in cattle). The disease is worldwide but is missed easily as it can mimic conditions like malignancy and tuberculosis. ${ }^{3}$ Predisposing factors include rural tropical areas, occupation like agricultural workers and poor dental hygiene. It can occur in any age with males being more commonly affected. The most common causative organism is Actinomyces israelii. Actinomycetes are bacteria producing filamentous branching hyphae that are normal inhabitants of human mouth. The main clinical variants include cervico-facial, thoracic, abdominal, primary cutaneous, pelvic and other rare variants like CNS, musculoskeletal and disseminated. Primary cutaneous variant is very uncommon, usually occurring on exposed skin as subcutaneous nodules that extend slowly and breakdown to form sinuses. Regional lymph nodes can be affected.

Pathological changes in the tissue include formation of granular colonies from which delicate mycelial filaments radiate (ray fungus). Around the colonies, a chronic neutrophilic \& lymphocytic infiltrate is noted, which tends to extend towards the epidermis, irrespective of where the primary lesion is. Gram positive filamentous organisms and sulphur granules on histological examination is strongly supportive of the diagnosis. Sulphur granules are not recovered in all cases and they are not specific for actinomycosis, but also seen in other conditions like nocardiosis, botryomycosis, etc. These granules are not actually composed of sulphur but are named so for its appearance and are actually filled with progeny bacteria that appear as round or oval basophilic masses with eosinophilic terminal clubs and can exhibit Splendore - Hoeppli phenomenon.

Direct isolation of the organism can be done but the failure rate of isolation is high (> $50 \%) .{ }^{4}$ Gram staining of pus and pathology of infected tissue is of great interest for diagnosis as it is usually more sensitive than culture. It is a chronic disease with marked fibrotic reaction \& therefore it is difficult to achieve the effective drug levels in the affected tissue. Hence a quick response to treatment should not be expected. It is sensitive to the antibiotics used in treating gram - positive bacterial infections. It is extremely susceptible to beta lactams, especially penicillin G or amoxicillin \& hence considered as drug of choice. ${ }^{5}$ Sulphonamides like sulfamethoxazole may also be used as an alternative at a dosage of $2-4$ gms per day. Since the response to therapy is slow and may take months, antibiotics are given usually for 6 12 months. But when combined with surgical drainage wherever possible (like in orocervicofacial disease), antibiotics may be given for a shorter course of 6 weeks. 


\section{FINAL DIAGNOSIS}

Primary Cutaneous Actinomycosis with Lymphadenitis

\section{CONCLUSIONS}

Actinomycosis is a rare chronic disease which can mimic malignancy and physicians have to be aware of the typical presentations. Bacterial culture and histopathology are the cornerstones for diagnosis. There are no specific guidelines for the duration of antibiotic therapy. With an increasing number of case reports of actinomycosis, standardized treatment regimens with shorter durations of therapy may be developed to help in appropriate management of the patients.

Informed consent was obtained from the patient.

Financial or Other Competing Interests: None.

\section{REFERENCES}

[1] Sullivan DC, Chapman SW. Bacteria that masquerade as fungi: actinomycosis/nocardia. Proc Am Thorac Soc 2010; $7(3): 216-21$.

[2] Veenakumari L, Sridevi C. Actinomycosis in histopathology-review of literature. International Archives of Integrated Medicine 2017;4(9):195-206.

[3] Acevedo F, Baudrand R, Letelier LM, et al. Actinomycosis: a great pretender. Case reports of unusual presentations and a review of the literature. Int $\mathrm{J}$ Infect Dis 2008;12(4):358-62.

[4] Wong VK, Turmezei TD, Weston VC. Actinomycosis. BM] 2011;343:d6099.

[5] Valour F, Sénéchal A, Dupieux C, et al. Actinomycosis: etiology, clinical features, diagnosis, treatment, and management. Infect Drug Resist 2014;7:183-97. 